\title{
Origen alemán de la comunidad terapéutica
}

Fernando Pérez del Río. Doctor en Psicólogia. Proyecto Hombre Burgos. Burgos.

Correspondencia: C/ Pedro Poveda Castroverde No 3, Bajo. 09007

947484770

proyectohombreburgos@sarenet.es

Palabras clave: Comunidad Terapéutica, historia, drogas, drogodependientes, sanatorio.

\section{Resumen:}

Se expone la posibilidad de poder ubicar el origen de lo que entendemos hoy en día por Comunidad Terapeuta en Alemania, concretamente en Tegel Berlín.

\section{GERMAN ORIGIN OF THE THERAPEUTIC COMMUNITY}

Keywords: therapeutic community, history, drugs, drug addicts, sanatorium

\section{Summary:}

The text presents the possibility of situating the origin of what we understand today as the therapeutic community in Germany, more specifically in Tegel, Berlin.

Si nos propusiéramos realizar una extensa revisión en el tiempo sobre el concepto de "comunidad", podríamos llegar a retrotraernos fácilmente a las primeras órdenes religiosas, "comunidades precristianas como la llamada Quamran", (1), o los Kibbutz en Israel. Terapéutica proviene del griego "therapeutikos", que significa cambio, y "comunidad" se define como "aquel grupo de personas que se unen para alcanzar uno o varios objetivos que por sí solas no podrían o les sería difícil cumplir" (2). Pero no entra en nuestro propósito extendernos demasiado, así que nos centraremos exclusivamente en las Comunidades Terapéuticas para drogodependientes.

Las primeras Comunidades Terapéuticas semidirectivas democráticas para el tratamiento de las adicciones se diseñaron y tuvieron su arranque en 
el continente europeo. En su origen eran "medio-sanatorios", ya que estaban ubicados en espacios hospitalarios.

Según psiquiatra francés Rogues de Fursac en su Manual de Psiquiatría del año 1921, (3) "el asilo de alcohólicos (o asilo de bebedores) propiamente dicho, debe ser un establecimiento de tratamiento y redención moral. Recibirá todos los sujetos susceptibles de enmienda. [...] El medio más eficaz es meterlos en las sociedades de abstinencia, donde encuentran ayuda, protección, vigilancia y vida social."

Rogues de Fursac, entre otras cosas médico jefe de los asilos del sena, expone en 1921 como las medidas preconizadas por la Comisión sueca: La lutte antialcoolique dans les Pays scandinaves, (acción directa e individual sobre el bebedor), tuvieron una influencia importante $\mathrm{y}$, por otro lado destaca la importancia de ubicar en establecimientos especiales a los reincidentes e incorregibles; disponerlos en lugares donde pudieran realizar diferentes tareas por ejemplo agrícolas, de tal modo que su manutención no ocasionara demasiado gasto a la sociedad. Pero en relación a este último punto, subraya a modo de conclusión satisfactoria, que estas experiencias de asilos para bebedores ya estaban realizándose concretamente en Alemania desde hace años, al parecer con buenos resultados.

Los clínicos alemanes del siglo XIX iban tanteando diferentes formas de actuar, y algunos observaron la importancia de implantar un cuidado especial para gente muy deteriorada. Todo esto fue desembocando en la particular propuesta del neurólogo y psicoanalista alemán Ernst Simmel en los años veinte del siglo XX. Este clínico creó un modelo terapéutico en Tegel (Berlín), donde el personal informaba de los actos de los pacientes adictos (4). "Para Simmel (Psychoalalitic treatment in a sanatorium, 1929) (5), se trataba esencialmente de reunir los distintos aspectos de la transferencia en una única y central que él pudiera interpretar sin limitación. Paralelamente, se restringía expresamente la vida pulsional de los pacientes, es decir, nada de relaciones sexuales, prohibición de consumir alcohol, dietas estrictas y otras limitaciones análogas. Cabe mencionar que, en sus inicios, este tipo de tratamiento estaba especialmente dirigido a pacientes toxicómanos" (6).

Podemos destacar también otras aportaciones e ideas sobre el tratamiento para adictos (Simmel, 1937), sistema que el autor calificó en su día de metapsicología: a) en primer lugar, se precisa tratar al paciente in situ, conviviendo en la estructura diseñada para tal efecto; b) el personal trabaja en equipo, concibiendo propuestas y elaborando conjuntamente los planes individualizados; de este modo, todo el equipo conocía los objetivos y desarrollo de cada paciente; c) su estructura y forma de trabajar confiaba en los recursos de las personas, en su fuerza, en que ellos fueran realizando un trabajo en interacción: un trabajo interpersonal. Como consecuencia, el Centro se constituye como lugar de entrenamiento donde los pacientes van regulando su conducta y donde se van encontrando salidas aceptables a sus tendencias; d) se trabajaba el contexto familiar y los roles familiares si el caso lo precisaba; e) se trata de un sistema donde -ya en los años veinte- 
se abordó el objetivo de que los terapeutas expertos formasen a los nuevos. Exactamente tal y como se hace hoy día. Sin duda, lo puesto en práctica por Simmel nos recuerda a lo que hacemos hoy en día; cierto es también que otras cuestiones como, por ejemplo, el importante concepto de autoayuda entre los pacientes, no fueron explicitadas por Simmel en sus textos.

En otro orden de cosas y atendiendo a la tradición de la psiquiatría del siglo XIX, vemos que ya existía en Alemania una orientación de corte más psicológica y pionera en Europa (die Psychiker) frente al movimiento organicista llamado die Somatiker. También encontramos autores como Johann Christian Heinroth (1773-1843) y, sobre todo, Griesinger (18171868); ambos influirían enormemente en la psiquiatría europea. Así no resulta extraño que surgieran originales propuestas provenientes de clínicos alemanes.

Pero estas precursoras experiencias comunitarias desarrolladas en Alemania no tardaron en caer en el más profundo de los olvidos. De hecho, abundantes textos fueron perseguidos e incluso quemados por el partido que gobernó Alemania antes de la II Guerra Mundial, según narra el propio Ernst Simmel (1882-1947), quien por cierto provenía de una familia judía y fue detenido por la Gestapo durante un tiempo por ser miembro de la Asociación de Médicos Socialistas; logró escapar a Bélgica, después huyó al Reino Unido y finalmente terminó en Estados Unidos ejerciendo como clínico. Sea como fuere, estas propuestas no aparecen citadas prácticamente en casi ningún libro, salvo muy honrosas excepciones. Para mayor inri, lo que sí encontramos en casi la mayoría de las publicaciones son comentarios como los siguientes: "El término Comunidad Terapéutica es moderno. Fue utilizado por primera vez para describir las comunidades terapéuticas psiquiátricas que nacieron en el Reino Unido durante los años 40." [...] "Comunidades Terapéuticas en hospitales psiquiátricos, propuestas y llevadas a la práctica por Jones (1953) y otros profesionales pioneros en el Reino Unido" (7).

El autor, De Leon, considerado "gurú" en la materia, llega a sostener lo siguiente: "La Comunidad Terapéutica psiquiátrica prototipo fue desarrollada por primera vez en la unidad de rehabilitación social del Hospital Belmont (llamado posteriormente Henderson), en Inglaterra, a mediados de los años 40. [...] Y la gran novedad consistía en tratar a pacientes internos psiquiátricos con trastornos de personalidad a largo plazo". En definitiva, y como el lector ya habrá advertido, esta "gran novedad" clínica se desarrolló en Inglaterra décadas después de la experiencia de Tegel en Berlín. El origen de la Comunidad Terapéutica, pues, se establece "oficialmente" en una unidad de rehabilitación para pacientes con trastornos "de personalidad", es decir, que ni siquiera eran drogodependientes. En resumidas cuentas, resulta difícil entender por qué se ignoran las definitivas y cuantiosas aportaciones germánicas con relación a la Comunidad Terapéutica.

En lo que respecta a España, recientemente fue publicado un libro bajo el título de Historia de las adicciones y su abordaje en España, editado por 
la asociación científica Sociodrogalcohol, donde se nos recuerda muy por encima y sin mayor lujo de detalles que el movimiento de las Comunidades Terapéuticas, como alternativa a la asistencia hospitalaria para los trastornos adictivos, tuvo su cuna en EE.UU. Y en cuanto a Europa atañe, en 1972 Lucien Engelmeier habría fundado la organización "Le Patriarche" ( $L a$ Boere, Francia), un modelo asistencial en régimen libre de drogas (8). En fin...

No obstante todo lo cual, se nos ocurre pensar que tal vez la razón principal por la cual no se reconozca el origen germánico de la Comunidad Terapéutica sea que el pueblo alemán perdió la II Guerra Mundial. Otra razón a sumar que podemos esbozar es la lucha entre modelos teóricos. Concretamente, este autor del que hablamos, Ernst Simmel, era psicoanalista. No todas las personas afiliadas a un modelo teórico concreto están emocionalmente preparadas para digerir que un modelo con fama de "individualista" sea el promotor de algo tan grupal como la Comunidad Terapéutica.

Llegados a este punto, habremos de detenernos un instante en otra posible causa que explique este tenaz ninguneo a la aportación teutona. $\mathrm{Si}$ se nos permite poner como ejemplo a "George de León", podría ser simplemente que este autor anglosajón no tenga a bien citar a autores que no hablan o escriben en otro idioma que no sea el inglés. Otra posible razón que podemos aventurar es que el terapeuta se forma en las propias comunidades terapéuticas, y durante muchos años -casi como si se tratara de una tradición- no se ha insertado la cultura de registrar de forma escrita este saber. Tanto la metodología como los instrumentos, y sobre todo las formas de actuar con los pacientes en diferentes situaciones, se trasmiten casi siempre oralmente, de una forma muy práctica. Son estilos que ayudan a un terapeuta a enfrentarse a situaciones con un fuerte componente emocional. En una expulsión, por ejemplo, un terapeuta fácilmente puede experimentar intenso miedo, y trabajar y entender esas emociones propias es algo difícil de ser plasmado sobre un papel. Se aprende fundamentalmente de la experiencia, del ensayo-error, pero sobre todo de la imitación del estilo y de las habilidades del compañero con más experiencia al que se le atribuye un supuesto saber. Con todo esto se desea indicar que lo que hoy conocemos mejor es lo que posiblemente más cerca de nosotros ha acontecido en el tiempo, quedando en el olvido todo lo demás.

En definitiva, podemos aseverar sin gran riesgo a equivocarnos que no existió una fuerte cultura a la hora de registrar la historia, salvo en estas últimas décadas. Tampoco ha sido frecuente verificar las fuentes de información ni comprobar si estas recetas históricas que nos indican de donde venimos son ciertas o no. Es decir, padecemos un problema del que se quejan no pocos historiadores.

No es de extrañar que en uno de los últimos libros publicados sobre comunidades terapéuticas en España, su autor, el sociólogo Domingo Comas (2005) (9) utilizara en su introducción la siguiente cita de Marco Fabio 
Quintiliano: "si la memoria no se ejercita, desaparece el recuerdo de las cosas, de tal manera que, cuando falta el recuerdo, la cosa deja de existir, aunque sea tan real como una piedra en el camino"

La conclusión de todo esto parece obvia: el origen de la Comunidad Terapéutica para drogodependientes es difícilmente atribuible a un momento histórico concreto, pero, dado el material expuesto, vemos que el hecho de proponer el origen de la Comunidad puede responder en ciertos casos, más, a diversos intereses personales de algunos autores que al rigor histórico. Quizás ya sea tiempo, en pleno siglo XXI como estamos, de poner a cada uno en su sitio; reconocer las definitivas aportaciones de los alemanes a la Comunidad Terapéutica y concretamente las contribuciones de Ernst Simmel (1882-1947), y hacer un poquito de justicia.

\section{Bibliografía.}

(1) Harris, M., (1980). Vacas, cerdos, guerras y brujas. Madrid: Alianza Editorial.

(2) Palacios, J. (2004). Ética y comunidad terapéutica: en búsqueda de una autocrítica. En: Salas, L. Desafíos y avances en la prevención y el tratamiento de las drogodependencias. Madrid: Proyecto Hombre. P 197206.

(3) De Fursac, R. (1921). Manual de Psiquiatría. Valencia: Editorial Pubul.

(4) Pérez, F. Martín, I. (2007). Nuevas Adicciones ¿Adicicones Nuevas? Guadalajara: Ediciones Intermedio.

(5) Simmel, E. (1937). The psychoanalytic sanitarium and the psychoanalytic movement, Bulleting of the Menninger Clinic, 1, 133-143.

(6) Álvarez, J. M., Esteban, R., Sauvagnat, F. (2004). Fundamentos de psicopatología psicoanalítica. Madrid: Síntesis.

(7) De Leon, G. (2004). La comunidad terapéutica y las adicciones. Teoría, modelo y método. Bilbao: Desclée De Brouwer.

(8) Santodomingo, F. (2009). Antecedentes históricos de las Adicciones. Del siglo XIX hasta 1940. (Coord.) Torres, A. Historia de las adicciones en la España contemporánea. Ed sociodrogalcohol.

(9) Comas, D. (2006). Comunidades Terapéuticas en España. Situación actual y propuesta funcional. Madrid: Grupo GID. 